\title{
THE SPORT EDUCATION MODEL IN ELEMENTARY AND SECONDARY EDUCATION: A SYSTEMATIC REVIEW
}

\author{
O MODELO DE SPORT EDUCATION NA EDUCAÇÃO PRIMÁRIA E \\ SECUNDÁRIA: REVISÃO SISTEMÁTICA
}

\author{
EL MODELO DE SPORT EDUCATION EN LA EDUCACIONN PRIMARIA Y \\ SECUNDARIA: REVISIÓN SISTEMÁTICA
}

\section{Carlos Evangelio*, Jacob Sierra-Díaz*, Sixto González-Víllora* Javier Fernández-Río**}

\section{Keywords: \\ Models, Educational. Physical Education and Training. Education, Primary and Secondary.}

\begin{abstract}
This study is an updated review of the most recent implementations of the Sport Education Model (SEM) from 1st to 12th grades (ages 6-18), including the newest research trends for future studies. A systematic review conducted from 2013 to 2017 used the main reference databases and original articles including information about instances of SEM implementation in any school grade. Results showed that SEM implementation has expanded enormously over the last five years to include all learning domains: physical, social, cognitive, and affective. However, such implementation is not easy and it demands specific teacher training. SEM seems to be a proper pedagogical approach for sports practice while developing motor skills, tactical-technical knowledge, and values.
\end{abstract}

Palavras chave

Modelos

educacionais.

Educação Física e

treinamento.

Ensino fundamental e médio.

Palabras clave: Modelos

Educacionales.

Educación Física

y Entrenamiento.

Educación Primaria

y Secundaria.
Resumo: Este estudo consistiu em oferecer uma revisão atualizada das implementações mais recentes do Modelo de Sport Education (SEM) do primeiro ao 12 ano (6-18 anos), incluindo as mais novas tendências de pesquisa para estudos futuros. Uma revisão sistemática de 2013 a 2017 foi realizada utilizando os bancos de dados de maior referência e os artigos originais que incluíam informações sobre as implementações do SEM em qualquer grau escolar. A implementação do SEM expandiu-se enormemente nos últimos cinco anos para incluir todos os diferentes domínios de aprendizagem: físico, social, cognitivo e afetivo. Entretanto, a implementação do SEM não é fácil e precisa de formação específica de professores. O SEM parece ser uma abordagem pedagógica adequada para praticar esporte, ao mesmo tempo em que desenvolve habilidades motoras, conhecimento técnico-tático e valores.

Resumen: El objetivo del estudio fue ofrecer una revisión de las implementaciones más recientes del Modelo Sport Education (SEM), desde primero de Educación Primaria hasta segundo de Bachillerato (6-18 años), incluyendo las más nuevas tendencias de investigación para estudios futuros. Se realizó una revisión sistemática desde 2013 hasta 2017, usando las principales bases de datos y los artículos originales que incluían informaciones sobre las implementaciones del SEM en cualquier grado escolar. La implementación del SEM se ha expandido enormemente en los últimos cinco años para incluir a todos los dominios del aprendizaje: físico, social, cognitivo y afectivo. Sin embargo, dicha implementación no es fácil y requiere formación específica del profesorado. El SEM parece un abordaje pedagógico adecuado para practicar deporte, al mismo tiempo que desarrolla habilidades motoras, conocimiento técnico-táctico y valores.
*University of Castilla - La Mancha. Cuenca, España. E-mail: carlos.evangelio.92@gmail.com; jacobsierradiaz@hotmail.com; sixto.gonzalez@uclm.es

**University of Oviedo. Oviedo, España. E-mail: javier.fernandez@uniovi.es

Recebido em: 03-04-2018 Aprovado em: 25-08-2018

DOI: https://doi.org/10.22456/1982-8918.81689 (c) (1) ()ㅡㄴ Licence 


\section{INTRODUCTION}

Sport Education is a pedagogical model (SEM) designed to provide authentic sport experiences in Physical Education (PE), and to develop competent, literate and enthusiastic sportspersons (SIEDENTOP; HASTIE; VAN DER MARS, 2011). It is probably one of the most widely implemented and researched instructional approach all over the world. The goal of the current article is to review the existing SEM scientific literature, building on previous reviews (ARAÚJO; MESQUITA; HASTIE, 2014; HASTIE; MARTÍNEZ; CALDERÓN, 2011; HASTIE; WALLHEAD, 2016; WALLHEAD; O'SULLIVAN, 2005). There has been an exponential increase around the world (mainly in Portugal or Spain) of SEM studies from 2013 to 2017, written in English, Spanish or Portuguese, many of them not collected in previous reviews.

Wallhead and O'Sullivan (2005) built their review around the development of the five main aims of PE (ALEXANDER; LUCKMAN, 2001): motor skill development, tactical knowledge and performance, fitness, social development and student attitudes and values. Hastie, Martínez and Calderón (2011) organized their review around the main aims of PE: fitness, skill development, game play, tactical awareness, personal/social development, and students' attitudes and values. Araújo, Mesquita and Hastie (2014) structured their review around four ideas: the effects of time on the students' learning outcomes, the control of the teaching-learning process, the dynamics of the peer-teaching tasks and the content selected. Finally, Hastie and Wallhead (2016) shaped their review around students' competence, literacy and enthusiastic participation.

The present systematic review has been organized around SEM's impact on the four learning outcomes of PE: cognitive, social, affective and physical. These are considered legitimate learning outcomes of PE, and they should be addressed (KIRK, 2013). For all the above, the aim of this article was to provide an updated international systematic review on the SEM implementation from $1^{\text {st }}$ to $12^{\text {th }}$ grade (6-18 years; elementary, middle and high school) to find new trends of research.

\section{METHOD}

A systematic review has been conducted to summarize the available evidence around the SEM over the last five years. This type of review focus on the key elements of the specific studies that implemented the topic under review (SEM) to recap the existing information, and subsequently, analyse and compare the studies with similar ones (MANTEROLA et al., 2013).

SEM literature produced from 2013 to 2017 was searched systematically using seven electronic databases: EBSCO host, ERIC, Google Scholar, Medline, SCOPUS, SPORTDiscus and Web of Science; which included journal papers found in scientific journals related to sports and education. The following descriptors were used: "Sport Education (Model)", "(elementary, middle and high) school" and "young students". Additionally, both English Boolean data type "and" and "or" were used.

Initially, as it is showed in Figure 1, 6.856 publications were found using the mentioned keywords at the databases cited above. 4.317articles were excluded because: (I) they were duplicated; (II) they were not published in peer reviewed journals indexed in the Journal Citation Report (JCR) or the Scimago Journal Rank (SJR); and/or (III) they were not implemented in any school. 
Figure 1 -The flow diagram of the systematic review process.

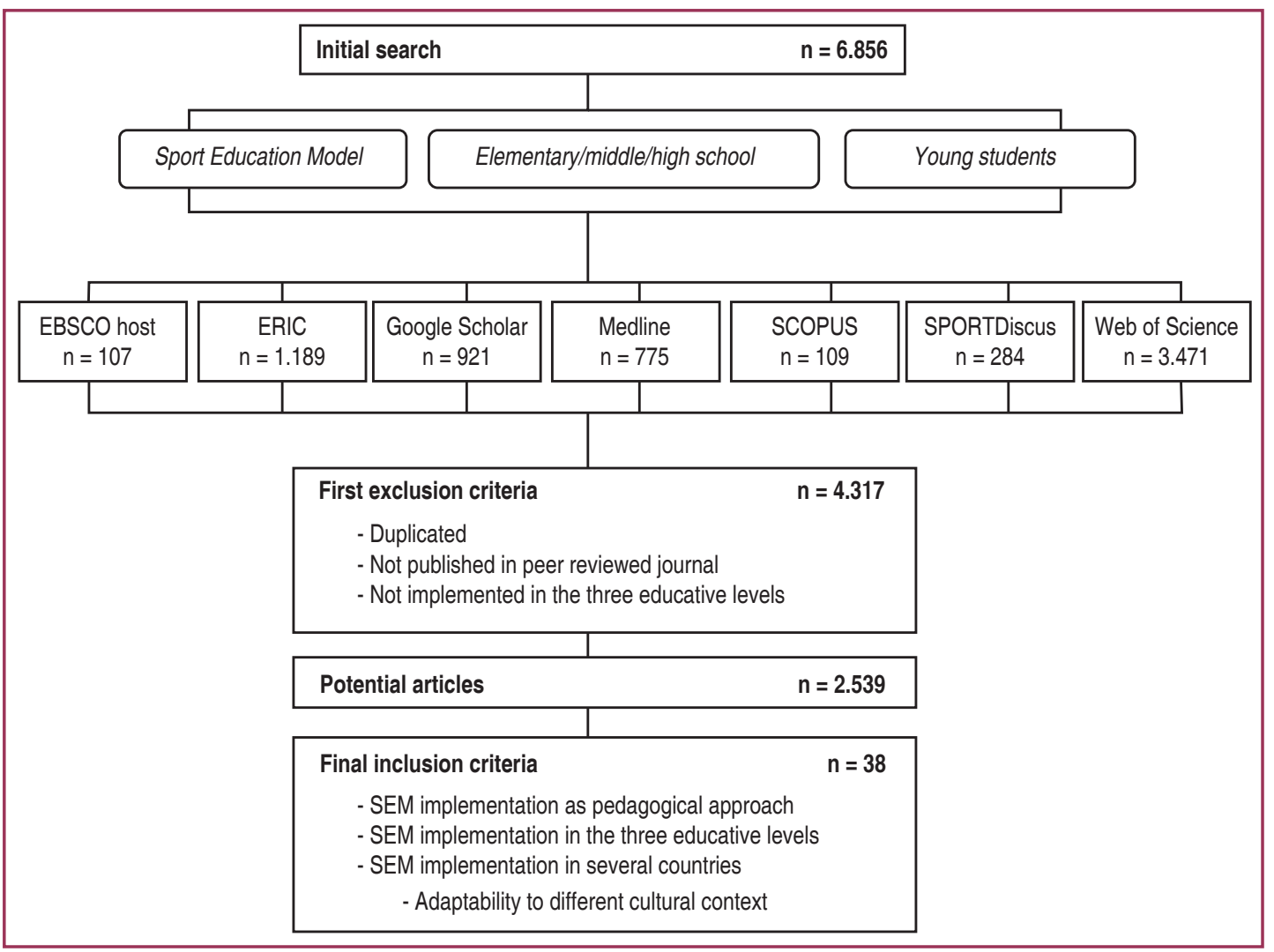

Source: prepared by authors.

Finally, 2.539 articles were considered potential studies, but only 38 were included according to the following criteria: (I) they implemented the SEM as a pedagogical approach; (II) they included information about SEM implementation in elementary ( $\left(^{\text {st }}\right.$ to $5^{\text {th }}$ grade -6 to 10 years), middle ( $6^{\text {th }}$ to $8^{\text {th }}$ grade -11 to 13 years $)$ and/or high school $\left(9^{\text {th }}\right.$ to $12^{\text {th }}$ grade -14 to 18 years); and (III) they included information about the SEM in several countries to observe the adaptations performed in the model based on the cultural and geographical context.

The summary of the 38 articles selected (Table 1) includes: author (s) and year of publication; country, grade (s) and length (s); content (s) and analysis (conducted); purpose (s) of the study; results; and learning outcomes.

\section{RESULTS AND DISCUSSION}

The results have been discussed around the key elements used to categorize the articles included in this review (country; students' grade/length; sport/content; analysis) and SEM' impact on the learning outcomes of PE (personal/social development: social and affective outcomes; game performance/tactical knowledge: cognitive and physical outcomes). Table 1 summarizes the studies included in the review from the last five years (2013-2017).

\subsection{Country}

Results showed that most published research was conducted in the USA, Spain and Portugal. However, new countries have emerged in this research field. SEM, which originated in the USA and later moved to Australia and Europe (HASTIE; MARTÍNEZ; CALDERÓN, 
2011; WALLHEAD; O'SULLIVAN, 2005), has expanded to countries such as Singapore (CHONG; PENNEY, 2013), Finland (ROMAR; SARÉN; HASTIE, 2016) and Brazil (GINCIENE; MATTHIESEN, 2017). This means that SEM can be implemented in many different educational contexts. However, some aspects of the model (i.e., number of sessions or roles performed by the students) had to be adapted to facilitate its implementation in different countries (GINCIENE; MATTHIESEN, 2017).

\subsection{Students' grade/ length}

Studies conducted in students of all elementary, middle and high school grades ( $11^{\text {st }}-12^{\text {th }}$; 6-18 years) were observed: $26,32 \%$ were conducted in several grades, $28,95 \%$ in elementary schools (most at $4^{\text {th }}-5^{\text {th }}$ grade), $26,32 \%$ in middle schools (most at $6^{\text {th }}$ grade), $26,32 \%$ in high schools (most at $10^{\text {th }}$ grade), and $18,42 \%$ at various educational levels. Results showed that SEM implementation was larger in elementary school $\left(4^{\text {th }}-5^{\text {th }}\right.$ grade), followed by $10^{\text {th }}$ grade of high school.

Previous reviews (HASTIE; MARTÍNEZ; CALDERÓN, 2011; ARAÚJO; MESQUITA; HASTIE, 2014) identified middle school and $6^{\text {th }}$ grade as the most frequent years, followed by high school, but only two studies (QUILL; CLARKE, 2005; CALDERÓN; HASTIE; MARTÍNEZ, 2010) focused on the early grades (earlier than $4^{\text {th }}$ grade). Over the last 4 years, there has been a shift in SEM implementation and assessment to elementary school. These years are considered a sensitive period in the individuals' development and game learning has been recommended (EVANGELIO et al., 2016).

The present review has showed that the SEM can be implemented in early grades in Spain and the USA (earlier than5 ${ }^{\text {th }}$ grade) (GUTIÉRREZ et al., 2014; LAYNE; HASTIE, 2014a; CALDERÓN et al., 2016; MARTÍNEZ; MÉNDEZ-GIMÉNEZ; VALVERDE, 2016). However, SEM implemented in very young students required greater teacher preparation (LAYNE; HASTIE, 2014a). Regarding the length of the studies, the majority lasted from 10 to 15 lessons $(47,36 \%)$, followed by interventions from 16 to 20 lessons (42,10\%). 12-lessons length was the most popular intervention framework $(21,05 \%)$. Finally, one implementation was conducted in only four lessons, other in six lessons, and two different studies were conducted in 20-25 and 28 lessons each.

Table 1 - Summary of studies on SEM in the last five years (2013-2017).

\begin{tabular}{|c|c|c|c|c|c|}
\hline $\begin{array}{l}\text { Author (s) } \\
\text { (Year) }\end{array}$ & $\begin{array}{c}\text { Country/ } \\
\text { grade (s)/ } \\
\text { length }\end{array}$ & $\begin{array}{c}\text { Content (s)/ } \\
\text { Analysis } \\
\text { (type) }\end{array}$ & Purpose (s) & Results & $\begin{array}{c}\text { Learning } \\
\text { Outcome } \\
\text { (s) }\end{array}$ \\
\hline $\begin{array}{c}\text { Araújo } \\
\text { et al. (2013) }\end{array}$ & $\begin{array}{l}\text { Portugal } \\
7^{\text {th }} \text { grade } \\
20 \text { lessons }\end{array}$ & Volleyball & $\begin{array}{l}\text { Assess the evolution } \\
\text { of content knowledge } \\
\text { from the teacher to } \\
\text { student-coaches in } \\
\text { a hybrid SEM-SGA } \\
\text { season. }\end{array}$ & $\begin{array}{l}\text { There was an evolution } \\
\text { on the student-coaches to } \\
\text { recognise the task but they } \\
\text { misunderstood the aims of } \\
\text { the tasks. }\end{array}$ & Cognitive \\
\hline $\begin{array}{c}\text { Calderón, } \\
\text { Martínez and } \\
\text { Hastie (2013) }\end{array}$ & $\begin{array}{l}\text { Spain } \\
5^{\text {th }} \text { grade }\end{array}$ & $\begin{array}{l}\text { Basketball, } \\
\text { hockey }\end{array}$ & $\begin{array}{l}\text { Compare SEM and } \\
\text { Direct Instruction } \\
\text { (DI) on students' } \\
\text { learning, implication } \\
\text { and motivation during } \\
\text { implementations. }\end{array}$ & $\begin{array}{l}\text { Students preferred SEM } \\
\text { because they became } \\
\text { more enthusiastic and } \\
\text { participative. They } \\
\text { improved their sport } \\
\text { knowledge, decision- } \\
\text { making and skills with both } \\
\text { models. }\end{array}$ & $\begin{array}{c}\text { Cognitive } \\
\text { and } \\
\text { physical }\end{array}$ \\
\hline
\end{tabular}


Table 1 continued...

\begin{tabular}{|c|c|c|c|c|c|}
\hline $\begin{array}{l}\text { Authors } \\
\text { (Year) }\end{array}$ & $\begin{array}{l}\text { Country/ } \\
\text { grade(s)/ } \\
\text { length }\end{array}$ & $\begin{array}{l}\text { Content (s)/ } \\
\text { Analysis } \\
\text { (type) }\end{array}$ & Purpose (s) & Results & $\begin{array}{l}\text { Learning } \\
\text { Outcome } \\
\text { (s) }\end{array}$ \\
\hline $\begin{array}{c}\text { Calderón, } \\
\text { Martínez and } \\
\text { Martínez (2013) }\end{array}$ & $\begin{array}{c}\text { Spain } \\
10^{\text {th }} \text { grade } \\
15 \text { lessons }\end{array}$ & $\begin{array}{c}\text { Qualitative and } \\
\text { quantitative }\end{array}$ & $\begin{array}{l}\text { Evaluate the effect } \\
\text { of the physical skill } \\
\text { perception on the } \\
\text { students' attitudes. }\end{array}$ & $\begin{array}{l}\text { Students and teacher } \\
\text { detected improvement in } \\
\text { physical skill perceptions } \\
\text { related to their implication } \\
\text { in the game and their } \\
\text { responsibility, specially, in } \\
\text { high-skilled students. }\end{array}$ & Social \\
\hline $\begin{array}{l}\text { Chong and } \\
\text { Penney } \\
\text { (2013) }\end{array}$ & $\begin{array}{l}\text { Singapore } \\
\text { Primary } \\
\text { School } \\
15 \text { lessons }\end{array}$ & $\begin{array}{l}\text { Ultimate } \\
\text { Qualitative and } \\
\text { quantitative }\end{array}$ & $\begin{array}{l}\text { Assess pupils' ability } \\
\text { to control failures } \\
\text { in PE, and the } \\
\text { development of social } \\
\text { and emotional skills. }\end{array}$ & $\begin{array}{l}\text { Positive social and } \\
\text { emotional learning to copy } \\
\text { with failure in PE. }\end{array}$ & Affective \\
\hline $\begin{array}{l}\text { Fernández- } \\
\text { Río, Méndez- } \\
\text { Giménez and } \\
\text { Méndez- } \\
\text { Alonso (2013) }\end{array}$ & $\begin{array}{c}\text { Spain } \\
8^{\text {th }}-12^{\text {th }} \\
\text { grades } \\
12 \text { lessons }\end{array}$ & Quantitative & $\begin{array}{l}\text { Determine effects of } \\
\text { three instructional } \\
\text { models (DI, SEM } \\
\text { and SEM-self-made } \\
\text { material) on students' } \\
\text { physical self-concept. }\end{array}$ & $\begin{array}{l}\text { SEM with self-made } \\
\text { materials increased } \\
\text { significantly students' } \\
\text { physical self-concept. }\end{array}$ & Affective \\
\hline $\begin{array}{c}\text { García-López } \\
\text { and Gutiérrez } \\
\text { (2013) }\end{array}$ & $\begin{array}{c}\text { Spain } \\
5^{\text {th }}, 6^{\text {th }}, 8^{\text {th }} \\
\text { grade } \\
18 \text { lessons }\end{array}$ & $\begin{array}{c}\text { Handball } \\
\text { Quantitative }\end{array}$ & $\begin{array}{l}\text { Assess the effects } \\
\text { of a SEM season on } \\
\text { students' empathy and } \\
\text { assertiveness. }\end{array}$ & $\begin{array}{l}\text { Increased students' } \\
\text { empathy and } \\
\text { aggressiveness. } \\
\text { Decreased assertiveness } \\
\text { and passivity. }\end{array}$ & Affective \\
\hline $\begin{array}{l}\text { Gutiérrez } \\
\text { et al. } \\
\text { (2013) }\end{array}$ & $\begin{array}{l}\text { Spain } \\
5^{\text {th }}-11^{\text {th }} \\
\text { grade } \\
\text { Different } \\
\text { lengths }\end{array}$ & $\begin{array}{l}\text { Handball, } \\
\text { basketball, } \\
\text { badminton, } \\
\text { dances. } \\
\text { Qualitativeand } \\
\text { quantitative }\end{array}$ & $\begin{array}{l}\text { Evaluate students' } \\
\text { perceptions on a SEM } \\
\text { season. }\end{array}$ & $\begin{array}{l}\text { Students perceived more } \\
\text { learning when they had } \\
\text { more playing time and } \\
\text { games. They were more } \\
\text { enthusiastic through } \\
\text { affiliation, festivity and the } \\
\text { final event. Higher level of } \\
\text { improvement in girls. }\end{array}$ & Physical \\
\hline $\begin{array}{c}\text { Hastie } \\
\text { et al. (2013) }\end{array}$ & $\begin{array}{l}\text { Portugal } \\
10^{\text {th }} \text { grade } \\
10 \text { lessons }\end{array}$ & $\begin{array}{l}\text { Qualitative and } \\
\text { quantitative }\end{array}$ & $\begin{array}{l}\text { Compare the effects } \\
\text { between DI and SEM } \\
\text { on skill and technical } \\
\text { performance, and in } \\
\text { content knowledge. }\end{array}$ & $\begin{array}{l}\text { Both instructional models } \\
\text { had improvements in } \\
\text { technique and skill } \\
\text { execution, with better } \\
\text { results for SEM. In content } \\
\text { knowledge, only SEM had } \\
\text { improvements. }\end{array}$ & $\begin{array}{l}\text { Cognitive } \\
\text { and } \\
\text { physical }\end{array}$ \\
\hline $\begin{array}{c}\text { Hastie, Farias } \\
\text { and Gutiérrez } \\
(2013)\end{array}$ & $\begin{array}{c}\text { USA, } \\
\text { Spain, } \\
\text { Portugal } \\
5^{\text {th }}-6^{\text {th }} \text { grade } \\
18-20 \\
\text { lessons }\end{array}$ & $\begin{array}{l}\text { Handball } \\
\text { Qualitative }\end{array}$ & $\begin{array}{l}\text { Assess students and } \\
\text { teachers' reactions } \\
\text { and perceptions on a } \\
\text { SEM season. }\end{array}$ & $\begin{array}{c}\text { SEM provided } \\
\text { opportunities to develop } \\
\text { international relations } \\
\text { and increased students' } \\
\text { motivation. }\end{array}$ & Social \\
\hline $\begin{array}{l}\text { Layne and } \\
\text { Hastie } \\
\text { (2013) }\end{array}$ & $\begin{array}{c}\text { USA } \\
4^{\text {th }} \text { grade } \\
13 \text { lessons }\end{array}$ & Swirl ball & $\begin{array}{l}\text { Evaluate students' } \\
\text { ability to work } \\
\text { independently and } \\
\text { organize different } \\
\text { tasks related to the } \\
\text { SEM. }\end{array}$ & $\begin{array}{l}\text { Students were able to } \\
\text { work independently of } \\
\text { the teacher and achieve } \\
\text { the aims. They performed } \\
\text { instructional tasks and } \\
\text { managerial successfully. }\end{array}$ & Cognitive \\
\hline $\begin{array}{l}\text { Gutiérrez } \\
\text { et al.(2014) }\end{array}$ & $\begin{array}{c}\text { Spain } \\
2^{\text {nd }} \text { grade } \\
10 \text { lessons }\end{array}$ & $\begin{array}{l}\text { Qualitative and } \\
\text { quantitative }\end{array}$ & $\begin{array}{c}\text { Assess students and } \\
\text { teachers' perceptions } \\
\text { on the SEM. }\end{array}$ & $\begin{array}{l}\text { Students learned SEM } \\
\text { features and phases. } \\
\text { It demanded greater } \\
\text { implication from the } \\
\text { teachers, specially, at the } \\
\text { beginning of the season. }\end{array}$ & Cognitive \\
\hline
\end{tabular}


Table 1 continued...

\begin{tabular}{|c|c|c|c|c|c|}
\hline $\begin{array}{l}\text { Authors } \\
\text { (Year) }\end{array}$ & $\begin{array}{l}\text { Country/ } \\
\text { grade (s)/ } \\
\text { length }\end{array}$ & $\begin{array}{l}\text { Content (s)/ } \\
\text { Analysis } \\
\text { (type) }\end{array}$ & Purpose (s) & Results & $\begin{array}{l}\text { Learning } \\
\text { Outcome } \\
\text { (s) }\end{array}$ \\
\hline $\begin{array}{l}\text { Hastie } \\
\text { et al. } \\
\text { (2014) }\end{array}$ & $\begin{array}{l}\text { USA } \\
10^{\text {th }} \text { grade } \\
12 \text { lessons }\end{array}$ & $\begin{array}{l}\text { Qualitativeand } \\
\text { quantitative }\end{array}$ & $\begin{array}{l}\text { Develop a SEM } \\
\text { season designed to } \\
\text { create a mastery- } \\
\text { involving class climate } \\
\text { and assess students' } \\
\text { perceptions of the } \\
\text { importance of this } \\
\text { motivational climate. }\end{array}$ & $\begin{array}{c}\text { Students showed a } \\
\text { consistent perception of a } \\
\text { mastery climate. Teacher } \\
\text { used performance- } \\
\text { based task structures } \\
\text { and competition to get a } \\
\text { mastery climate based on } \\
\text { recognition and evaluation. }\end{array}$ & Cognitive \\
\hline $\begin{array}{l}\text { Layne and } \\
\text { Hastie } \\
(2014 a)\end{array}$ & $\begin{array}{c}\text { USA } \\
2^{\text {nd }} \text { grade }\end{array}$ & $\begin{array}{l}\text { Throwing, } \\
\text { and kicking at } \\
\text { targets }\end{array}$ & $\begin{array}{l}\text { Examine students, } \\
\text { teachers and } \\
\text { non-participants' } \\
\text { perceptions of a SEM } \\
\text { season. }\end{array}$ & $\begin{array}{l}\text { SEM could be applied in } \\
\text { lower levels of primary and } \\
\text { can help improve students' } \\
\text { learning. It is required } \\
\text { more teacher preparation } \\
\text { at the season start and } \\
\text { at the beginning of SEM } \\
\text { phases. }\end{array}$ & Cognitive \\
\hline $\begin{array}{l}\text { Layne and } \\
\text { Hastie } \\
\text { (2014b) }\end{array}$ & $\begin{array}{c}\text { USA } \\
4^{\text {th }} \text { grade } \\
13 \text { lessons }\end{array}$ & $\begin{array}{l}\text { Swirl ball } \\
\text { Qualitative and } \\
\text { quantitative }\end{array}$ & $\begin{array}{c}\text { Assess students' } \\
\text { ability to improve } \\
\text { game play capabilities. }\end{array}$ & $\begin{array}{l}\text { Students improved game } \\
\text { performance: the length of } \\
\text { the season provided the } \\
\text { framework for students to } \\
\text { improve skill competency. }\end{array}$ & Physical \\
\hline $\begin{array}{c}\text { Cuevas, } \\
\text { García-López } \\
\text { and Contreras } \\
(2015)\end{array}$ & $\begin{array}{c}\text { Spain } \\
10^{\text {th }} \text { grade } \\
19 \text { lessons }\end{array}$ & $\begin{array}{l}\text { Volleyball } \\
\text { Quantitative }\end{array}$ & $\begin{array}{l}\text { Evaluate students' } \\
\text { basic psychological } \\
\text { needs. }\end{array}$ & $\begin{array}{l}\text { Students significantly } \\
\text { improved competence. }\end{array}$ & Affective \\
\hline $\begin{array}{c}\text { Farias, } \\
\text { Mesquita and } \\
\text { Hastie (2015) }\end{array}$ & $\begin{array}{l}\text { Portugal } \\
5^{\text {th }} \text { grade } \\
17 \text { lessons }\end{array}$ & $\begin{array}{c}\text { Soccer } \\
\text { Qualitative and } \\
\text { quantitative }\end{array}$ & $\begin{array}{l}\text { Examine students' } \\
\text { understanding and } \\
\text { game performance in } \\
\text { a hybrid SEM-IGCM } \\
\text { program. } \\
\end{array}$ & $\begin{array}{l}\text { Positive impact on } \\
\text { students' game } \\
\text { performance and } \\
\text { understanding. }\end{array}$ & $\begin{array}{l}\text { Cognitive } \\
\text { and } \\
\text { physical }\end{array}$ \\
\hline $\begin{array}{l}\text { Mahedero } \\
\text { et al. (2015) }\end{array}$ & $\begin{array}{c}\text { Spain } \\
8^{\text {th }} \text { grade } \\
12 \text { lessons }\end{array}$ & $\begin{array}{l}\text { Mini- volleyball } \\
\text { Qualitative and } \\
\text { quantitative }\end{array}$ & $\begin{array}{c}\text { Examine performance } \\
\text { outcomes and game } \\
\text { knowledge. }\end{array}$ & $\begin{array}{l}\text { Medium skill level students } \\
\text { had bigger improvements } \\
\text { than high and low skilled } \\
\text { students in game play, skill } \\
\text { execution and knowledge. }\end{array}$ & $\begin{array}{l}\text { Cognitive } \\
\text { and } \\
\text { physical }\end{array}$ \\
\hline $\begin{array}{l}\text { Méndez- } \\
\text { Giménez, } \\
\text { Fernández-Río } \\
\text { and Méndez- } \\
\text { Alonso } \\
\text { (2015) }\end{array}$ & $\begin{array}{l}\text { Spain } \\
7^{\text {th }}-12^{\text {th }} \\
\text { grades }\end{array}$ & Quantitative & $\begin{array}{l}\text { Compare the effects } \\
\text { of DI, SEM and } \\
\text { SEM with self-made } \\
\text { materials on students' } \\
\text { sportsmanship, } \\
\text { friendship, basic } \\
\text { psychological needs } \\
\text { and achievement } \\
\text { goals. }\end{array}$ & $\begin{array}{l}\text { Significant improvements } \\
\text { in sportsmanship, basic } \\
\text { psychological needs, } \\
\text { friendship goals and } \\
\text { performance-avoidance } \\
\text { goals with SEM and SEM } \\
\text { with self-made materials. }\end{array}$ & $\begin{array}{l}\text { Social } \\
\text { and } \\
\text { affective }\end{array}$ \\
\hline $\begin{array}{l}\text { Pereira } \\
\text { et al. } \\
(2015)\end{array}$ & $\begin{array}{l}\text { Portugal } \\
6^{\text {th }} \text { grade } \\
20 \text { lessons }\end{array}$ & $\begin{array}{l}\text { Qualitativeand } \\
\text { quantitative }\end{array}$ & $\begin{array}{l}\text { Compare students' } \\
\text { technical performance } \\
\text { improvements in SEM } \\
\text { and DI. }\end{array}$ & $\begin{array}{l}\text { Boys and girls of all skill } \\
\text { levels improved in all } \\
\text { events with SEM, but only } \\
\text { high-level males improved } \\
\text { with DI for the approach } \\
\text { features. }\end{array}$ & Physical \\
\hline $\begin{array}{l}\text { Calderón } \\
\text { et al. } \\
(2016)\end{array}$ & $\begin{array}{l}\text { Spain } \\
3^{\text {rd }}-6^{\text {th }} \\
\text { grade } \\
10 \text { lessons }\end{array}$ & $\begin{array}{c}\text { Handball, } \\
\text { pichiQualitative } \\
\text { and } \\
\text { quantitative }\end{array}$ & $\begin{array}{l}\text { Assess the effects } \\
\text { of team-teaching } \\
\text { (co-teaching) to create } \\
\text { effective learning } \\
\text { backgrounds using the } \\
\text { SEM. }\end{array}$ & $\begin{array}{c}\text { Teamwork and } \\
\text { autonomy promoted } \\
\text { effective teacher-student } \\
\text { communication. Increased } \\
\text { positive social climate. Co- } \\
\text { assessment was another } \\
\text { important factor. }\end{array}$ & Social \\
\hline
\end{tabular}


Table 1 continued...

\begin{tabular}{|c|c|c|c|c|c|}
\hline $\begin{array}{l}\text { Authors } \\
\text { (Year) }\end{array}$ & $\begin{array}{l}\text { Country/ } \\
\text { grade (s)/ } \\
\text { length }\end{array}$ & $\begin{array}{l}\text { Content (s)/ } \\
\text { Analysis } \\
\text { (type) }\end{array}$ & Purpose (s) & Results & $\begin{array}{l}\text { Learning } \\
\text { Outcome } \\
\text { (s) }\end{array}$ \\
\hline $\begin{array}{l}\text { Cuevas, } \\
\text { García-López } \\
\text { and Serra- } \\
\text { Olivares (2016) }\end{array}$ & $10^{\text {th }}$ grade & Volleyball & $\begin{array}{l}\text { Assess the impact of } \\
\text { SEM on motivational } \\
\text { regulation, basic } \\
\text { psychological } \\
\text { needs, satisfaction- } \\
\text { enjoyment, boredom } \\
\text { and intention to be } \\
\text { physically active. }\end{array}$ & $\begin{array}{l}\text { Positive and significant } \\
\text { impact on students' } \\
\text { intrinsic motivation. } \\
\text { Small improvements } \\
\text { were guessed for } \\
\text { satisfaction-enjoyment, } \\
\text { self-determination, identified } \\
\text { regulation, and competence. }\end{array}$ & Affective \\
\hline $\begin{array}{l}\text { Martínez, } \\
\text { Méndez- } \\
\text { Giménez and } \\
\text { Valverde } \\
\text { (2016) }\end{array}$ & $\begin{array}{l}\text { Spain } \\
3^{\text {rd }-4^{\text {th }}} \\
\text { grade } \\
10 \text { lessons }\end{array}$ & $\begin{array}{l}\text { Pichi, ultimate, } \\
\quad \text { indiaca } \\
\text { Qualitativeand } \\
\text { quantitative }\end{array}$ & $\begin{array}{l}\text { Examine the effects } \\
\text { of the SEM on the } \\
\text { classroom's social } \\
\text { climate, perceived } \\
\text { competence and } \\
\text { intention to be } \\
\text { physically active. }\end{array}$ & $\begin{array}{l}\text { Group cohesion encouraged } \\
\text { fair play and motivation. } \\
\text { Role-playing as a tool to } \\
\text { learn the game. Significant } \\
\text { impacts: social climate } \\
\text { perception and intention to } \\
\text { be physically active. }\end{array}$ & $\begin{array}{l}\text { Social } \\
\text { and } \\
\text { affective }\end{array}$ \\
\hline $\begin{array}{l}\text { Méndez- } \\
\text { Giménez, } \\
\text { Martínez and } \\
\text { Valverde } \\
\text { (2016) }\end{array}$ & $\begin{array}{l}\text { Spain } \\
3^{\text {rd }-4^{\text {th }}} \\
\text { grade } \\
20 \text { lessons }\end{array}$ & $\begin{array}{l}\text { Ultimate, } \\
\text { indiaca }\end{array}$ & $\begin{array}{l}\text { Compare teacher and } \\
\text { students' assessment } \\
\text { procedures between a } \\
\text { SEM/SEM-self-made } \\
\text { materials approach. }\end{array}$ & $\begin{array}{l}\text { A preference for self-made } \\
\text { materials was observed, } \\
\text { and a rise in physical } \\
\text { activity practise. Pupils } \\
\text { indicated that self-made } \\
\text { materials could improve } \\
\text { their skills. }\end{array}$ & $\begin{array}{l}\text { Affective } \\
\text { and } \\
\text { physical }\end{array}$ \\
\hline $\begin{array}{l}\text { Menéndez- } \\
\text { Santurio and } \\
\text { Fernández- } \\
\text { Río (2016a) }\end{array}$ & $\begin{array}{c}\text { Spain } \\
10^{\text {th }} \text { grade } \\
16 \text { lessons }\end{array}$ & $\begin{array}{l}\text { Kickboxing } \\
\text { Qualitative and } \\
\text { quantitative }\end{array}$ & $\begin{array}{l}\text { Introduce educational } \\
\text { kickboxing through } \\
\text { a hybrid SEM-TPSR } \\
\text { season. }\end{array}$ & $\begin{array}{l}\text { Kickboxing is an adequate } \\
\text { content for PE; the } \\
\text { hybridization helped develop } \\
\text { a student-centred context. }\end{array}$ & Cognitive \\
\hline $\begin{array}{l}\text { Menéndez- } \\
\text { Santurio and } \\
\text { Fernández- } \\
\text { Río (2016b) }\end{array}$ & $\begin{array}{c}\text { Spain } \\
12^{\text {th }} \text { grade }\end{array}$ & Quantitative & $\begin{array}{l}\text { Evaluate the effects of } \\
\text { a hybrid SEM-TPSR } \\
\text { season on students' } \\
\text { friendship goals, } \\
\text { responsibility, basic } \\
\text { psychological needs } \\
\text { and violence. }\end{array}$ & $\begin{array}{l}\text { The hybridization had } \\
\text { significant positive } \\
\text { improvements in the } \\
\text { relatedness, students' } \\
\text { violence, social } \\
\text { responsibility and } \\
\text { competence. }\end{array}$ & $\begin{array}{l}\text { Social } \\
\text { and } \\
\text { affective }\end{array}$ \\
\hline $\begin{array}{l}\text { Pereira } \\
\text { et al. } \\
(2016)\end{array}$ & $\begin{array}{l}\text { Portugal } \\
6^{\text {th }} \text { grade }\end{array}$ & $\begin{array}{l}\text { Qualitative and } \\
\text { quantitative }\end{array}$ & $\begin{array}{l}\text { Contrast the } \\
\text { effects of SEM and } \\
\text { DI on students' } \\
\text { content knowledge, } \\
\text { considering their sex } \\
\text { and skill level. }\end{array}$ & $\begin{array}{l}\text { Improvements: movement } \\
\text { patterns, task organization } \\
\text { and teacher' feedback in } \\
\text { Dl; affective and cognitive } \\
\text { implication, autonomy in } \\
\text { the task transitions and } \\
\text { taking decisions in SEM. }\end{array}$ & Affective \\
\hline $\begin{array}{l}\text { Romar, Sarén, } \\
\text { and Hastie } \\
(2016)\end{array}$ & $\begin{array}{l}\text { Finland } \\
6^{\text {th }} \text { grade } \\
11 \text { lessons }\end{array}$ & $\begin{array}{c}\text { Soccer } \\
\text { Qualitativeand } \\
\text { quantitative }\end{array}$ & $\begin{array}{l}\text { Examine students, } \\
\text { coaches and parents' } \\
\text { perceptions and } \\
\text { experiences on the } \\
\text { SEM. }\end{array}$ & $\begin{array}{l}\text { Students enjoyed rolesand } \\
\text { tasks in a student- } \\
\text { centred context. There } \\
\text { was a positive teams' } \\
\text { atmosphere. }\end{array}$ & Social \\
\hline $\begin{array}{l}\text { Wahl- } \\
\text { Alexander, } \\
\text { Sinelnikov and } \\
\text { Curtner-Smith } \\
\text { (2016) }\end{array}$ & $\begin{array}{c}\text { USA } \\
8^{\text {th }} \text { grade } \\
18-21 \\
\text { lessons }\end{array}$ & $\begin{array}{l}\text { Different team } \\
\text { sports }\end{array}$ & $\begin{array}{l}\text { Examine the } \\
\text { participation in a } \\
\text { significant number of } \\
\text { SEM seasons over a } \\
\text { period of five years. }\end{array}$ & $\begin{array}{l}\text { The students' prolonged } \\
\text { participation in SEM } \\
\text { seasons developed roles, } \\
\text { fair play and SEM goals: } \\
\text { enthusiastic, competent } \\
\text { and literate pupils. }\end{array}$ & $\begin{array}{l}\text { Social } \\
\text { and } \\
\text { cognitive }\end{array}$ \\
\hline $\begin{array}{l}\text { Araújo } \\
\text { et al. } \\
(2017)\end{array}$ & $\begin{array}{c}\text { Portugal } \\
7^{\text {th }}-9 \text { th } \\
\text { grade } \\
\text { Three } \\
\text { seasons (20- } \\
25 \text { lessons) }\end{array}$ & Qualitative & $\begin{array}{l}\text { Examine the evolution } \\
\text { of students and } \\
\text { coaches' Pedagogical } \\
\text { Content Knowledge } \\
\text { (PCK) with a SEM- } \\
\text { SGA hybridization. }\end{array}$ & $\begin{array}{l}\text { Interventions were } \\
\text { effective in developing } \\
\text { student-coaches' } \\
\text { PCK, allowing a more } \\
\text { complete transfer of } \\
\text { the responsibility to the } \\
\text { students. }\end{array}$ & $\begin{array}{l}\text { Cognitive } \\
\text { and social }\end{array}$ \\
\hline
\end{tabular}


Table 1 continued...

\begin{tabular}{|c|c|c|c|c|c|}
\hline $\begin{array}{c}\text { Authors } \\
\text { (Year) }\end{array}$ & $\begin{array}{l}\text { Country/ } \\
\text { grade (s)/ } \\
\text { length }\end{array}$ & $\begin{array}{l}\text { Content (s)/ } \\
\text { Analysis } \\
\text { (type) }\end{array}$ & Purpose (s) & Results & $\begin{array}{l}\text { Learning } \\
\text { Outcome } \\
\text { (s) }\end{array}$ \\
\hline $\begin{array}{l}\text { Fernández- } \\
\text { Río, Méndez- } \\
\text { Giménez and } \\
\text { Méndez- } \\
\text { Alonso (2017) }\end{array}$ & $\begin{array}{c}\text { Spain } \\
8^{\text {th }}-11^{\text {th }} \\
\text { grades } \\
12 \text { lessons }\end{array}$ & Quantitative & $\begin{array}{l}\text { Compare the effects } \\
\text { of two instructional } \\
\text { approaches (SEM } \\
\text { and DI), on students' } \\
\text { psychological } \\
\text { response. }\end{array}$ & $\begin{array}{l}\text { SEM significantly improved } \\
\text { intrinsic motivation, social } \\
\text { responsibility, cooperative } \\
\text { learning, boredom, } \\
\text { autonomy, relationship and } \\
\text { competence. }\end{array}$ & Social \\
\hline $\begin{array}{l}\text { Fernández-Río } \\
\text { and Menéndez- } \\
\text { Santurio } \\
\text { (2017) }\end{array}$ & $\begin{array}{c}\text { Spain } \\
9^{\text {th }} \text { grade } \\
16 \text { lessons }\end{array}$ & Kickboxing & $\begin{array}{l}\text { Assess students and } \\
\text { teachers' perceptions } \\
\text { of a hybrid SEM- } \\
\text { TPSR season. }\end{array}$ & $\begin{array}{c}\text { Students develop } \\
\text { enjoyment } \\
\text { cooperation,responsibility, } \\
\text { and increase their learning } \\
\text { for the autonomy and } \\
\text { roles. }\end{array}$ & Social \\
\hline $\begin{array}{l}\text { Ginciene and } \\
\text { Matthiesen } \\
(2017)\end{array}$ & $\begin{array}{l}\text { Brasil } \\
6^{\text {th }} \text { grade } \\
6 \text { lessons }\end{array}$ & $\begin{array}{c}\text { Track and field } \\
\text { Qualitative }\end{array}$ & $\begin{array}{l}\text { Examine the effects } \\
\text { of SEM with track and } \\
\text { field teaching, in the } \\
\text { Brazilian educative } \\
\text { context. }\end{array}$ & $\begin{array}{l}\text { SEM was an effective } \\
\text { tool to develop the } \\
\text { three dimensions of PE } \\
\text { contents: conceptual, } \\
\text { procedural and attitudinal. }\end{array}$ & $\begin{array}{l}\text { Cognitive } \\
\text { and } \\
\text { affective }\end{array}$ \\
\hline $\begin{array}{l}\text { Gil-Arias } \\
\text { et al. } \\
(2017)\end{array}$ & $\begin{array}{c}\text { Spain } \\
12^{\text {th }} \text { grade } \\
16 \text { lessons }\end{array}$ & $\begin{array}{l}\text { Volleyball, } \\
\text { ultimate } \\
\text { Quantitative }\end{array}$ & $\begin{array}{l}\text { Investigate the effect a } \\
\text { hybrid TGfU-SEMunit } \\
\text { vs. DI, on pupils' } \\
\text { motivation to engage } \\
\text { in PE. }\end{array}$ & $\begin{array}{l}\text { Students' autonomy, } \\
\text { competence and } \\
\text { enjoyment raised when } \\
\text { they were taught using the } \\
\text { hybrid model. }\end{array}$ & Social \\
\hline $\begin{array}{c}\text { Hastie, } \\
\text { Ward and } \\
\text { Brock(2017) }\end{array}$ & $\begin{array}{c}\text { USA } \\
4^{\text {th }} \text { grade }\end{array}$ & $\begin{array}{l}\text { Qualitative and } \\
\text { quantitative }\end{array}$ & $\begin{array}{l}\text { Assess whether using } \\
\text { rated competition } \\
\text { can rise students' } \\
\text { opportunities for game } \\
\text { involvement and } \\
\text { success rates. }\end{array}$ & $\begin{array}{c}\text { Regarding efficiency } \\
\text { and success, high- } \\
\text { skilled players showed } \\
\text { advantages against } \\
\text { low-skilled players. Girls } \\
\text { had less involvement than } \\
\text { boys. }\end{array}$ & Physical \\
\hline $\begin{array}{l}\text { Méndez- } \\
\text { Giménez and } \\
\text { Martínez } \\
(2017)\end{array}$ & $\begin{array}{c}\text { Spain } \\
6^{\text {th }} \text { grade } \\
12 \text { lessons }\end{array}$ & $\begin{array}{l}\text { Mime } \\
\text { Qualitative and } \\
\text { quantitative }\end{array}$ & $\begin{array}{l}\text { Assess students' } \\
\text { perceptions. }\end{array}$ & $\begin{array}{l}\text { Students' increased their } \\
\text { competence and sport } \\
\text { culture and highly valued } \\
\text { the model's main features. }\end{array}$ & Cognitive \\
\hline $\begin{array}{l}\text { Méndez- } \\
\text { Giménez, } \\
\text { Martínez and } \\
\text { Valverde } \\
\text { (2017) }\end{array}$ & $\begin{array}{l}\text { Spain } \\
6^{\text {th }} \text { grade }\end{array}$ & $\begin{array}{l}\text { Qualitative and } \\
\text { quantitative }\end{array}$ & $\begin{array}{l}\text { Examine the } \\
\text { effects on students' } \\
\text { emotional intelligence } \\
\text { and motivational } \\
\text { mediators. }\end{array}$ & $\begin{array}{c}\text { Students' increased } \\
\text { attention, clarity and repair } \\
\text { (emotional intelligence), } \\
\text { and autonomy competence } \\
\text { and relatedness (basic } \\
\text { psychological needs). }\end{array}$ & Cognitive \\
\hline $\begin{array}{l}\text { Menéndez- } \\
\text { Santurio and } \\
\text { Fernández-Río } \\
\text { (2017) }\end{array}$ & $\begin{array}{c}\text { Spain } \\
12^{\text {th }} \text { grade }\end{array}$ & Qualitative & $\begin{array}{l}\text { Explore the impact } \\
\text { of the combination } \\
\text { of SEM-TPSR } \\
\text { for learners with } \\
\text { disabilities. }\end{array}$ & $\begin{array}{l}\text { SE-TPSR promotes } \\
\text { educative inclusion } \\
\text { (increases learning } \\
\text { enjoyment and } \\
\text { membership): students' } \\
\text { role, cooperation, } \\
\text { transference } \\
\text { andfriendship. }\end{array}$ & Social \\
\hline $\begin{array}{l}\text { Ward } \\
\text { et al. } \\
(2017)\end{array}$ & $\begin{array}{c}\text { USA } \\
5^{\text {th }} \text { grade }\end{array}$ & Quantitative & $\begin{array}{l}\text { Assess if SEM } \\
\text { provides levels of } \\
\text { physical activity and } \\
\text { increase their fitness } \\
\text { knowledge and } \\
\text { attainment. }\end{array}$ & $\begin{array}{c}\text { Significant improvements } \\
\text { in knowledge and fitness } \\
\text { tests. } 54.5 \% \text { of the lesson } \\
\text { were at moderate-to- } \\
\text { vigorous physical activity } \\
\text { level. }\end{array}$ & $\begin{array}{l}\text { Cognitive } \\
\text { and } \\
\text { physical }\end{array}$ \\
\hline
\end{tabular}

Source: the authors. 


\subsection{Sport/content}

Results showed that $78,95 \%$ of studies included just one content/sport, while $21,05 \%$ used several sports. In accordance with previous reviews (HASTIE; MARTÍNEZ; CALDERÓN, 2011; ARAÚJO; MESQUITA; HASTIE, 2014), the present one showed a predominance of invasion games, probably because affiliation is an essential feature of SEM (SIEDENTOP; HASTIE; VAN DER MARS, 2011), including newly researched sports such as handball (GARCÍALÓPEZ; GUTIÉRREZ, 2013) or mini-handball (HASTIE; WARD; BROCK, 2017). However, an increase has been observed in the following sport categories: divided-court games such as indiaca (MÉNDEZ-GIMÉNEZ; MARTÍNEZ; VALVERDE, 2016) and badminton (CALDERÓN; MARTÍNEZ; MARTÍNEZ, 2013; GUTIÉRREZ et al., 2013); striking and fielding games such as modified baseball or "Pichi" (CALDERÓN et al., 2016; MARTÍNEZ; MÉNDEZ-GIMÉNEZ; VALVERDE, 2016); throwing and kicking at a target (LAYNE; HASTIE, 2014a); and movingtarget games such as dodgeball (GUTIÉRREZ et al., 2014). Other sports/activities such as track and field (GINCIENE; MATTHIESEN, 2017; HASTIE et al., 2013) showed a very low number of studies in previous reviews.

Finally, the inclusion of dance (GUTIÉRREZ et al., 2013), combat sports (MENÉNDEZSANTURIO; FERNÁNDEZ-RÍO, 2016a; 2016b), mime (MÉNDEZ-GIMÉNEZ; MARTÍNEZ, 2017) and cross-fit (WARD et al., 2017) constitutes an innovation in SEM research. Self-made materials were also a new element. Studies conducted with this type of resources showed that it increases students' participation and skills (MÉNDEZ-GIMÉNEZ; MARTíNEZ; VALVERDE, 2016), self-concept (FERNÁNDEZ-RíO;MÉNDEZ-GIMÉNEZ; MÉNDEZ-ALONSO, 2013) and basic psychological needs (MÉNDEZ-GIMÉNEZ; FERNÁNDEZ-RíO;MÉNDEZ-ALONSO, 2015).

\subsection{Analysis (type)}

The majority of the studies used a mixed methods research methodology $(47,36 \%)$, followed by quantitative or qualitative methods alone (26,31\% each one). Among the quantitative methods used to assess the SEM impact on the students there were performance tasks, tests or questionnaires such as the "Basic Psychological Needs in Exercise Scale" (VLACHOPOULOS; MICHAILIDOU, 2006) used by Gil-Arias et al. (2017), or the "Sport Education benchmark observation instrument" (SINELNIKOV, 2009) used by Romar, Sarén and Hastie (2016). Among the qualitative methods used, there were interviews (ARAÚJO et al., 2017), discussion groups (GUTIÉRREZ et al., 2013) or drawings (MÉNDEZ-GIMÉNEZ; MARTÍNEZ, 2017).

\subsection{Personal/social development: social and affective learning outcomes}

Results of the present review have showed the SEM' impact on social and affective learning outcomes, which connect with students' personal and social development (i.e., responsibility, social relationships, emotional learning, basic psychological needs, physical self-concept). Social learning outcomes were assessed in $21,05 \%$ of the studies, while affective learning outcomes in $18,42 \%$. Previous reviews indicated that SEM implementation can fulfil students' basic psychological needs: autonomy, competence and relatedness (MACPHAIL; KINCHIN, 2004), and new studies included in the present review supported this 
idea (FERNÁNDEZ-RÍO; MÉNDEZ-GIMÉNEZ; MÉNDEZ-ALONSO, 2017; MAHEDERO et al., 2015), including hybridizations between the SEM and Teaching for Personal and Social Responsibility (TPSR) (MENÉNDEZ-SANTURIO; FERNÁNDEZ-RÍO; 2016b).

The development of students' social skills (MÉNDEZ-GIMÉNEZ; FERNÁNDEZ-RíO; MÉNDEZ-ALONSO, 2015) and intrinsic motivation (CUEVAS; GARCÍA-LÓPEZ; SERRAOLIVARES, 2016; FERNÁNDEZ-RÍO; MÉNDEZ-GIMÉNEZ; MÉNDEZ-ALONSO, 2017) were also observed. Team affiliation has been considered a fundamental element in these improvements (PILL, 2008), because it can help promote positive communication, assertiveness, cooperation, empathy, autonomy and relationship among students (GARCÍA-LÓPEZ et al., 2012; GARCÍA-LÓPEZ; GUTIÉRREZ, 2013; MARTÍNEZ; MÉNDEZ-GIMÉNEZ; VALVERDE, 2016; ROMAR; SARÉN; HASTIE, 2016). The feeling of belonging to a team, as well as the cohesion that it creates, can also help improve students' fair play, motivation and enthusiasm (GARCÍA-LÓPEZ et al., 2012; WAHL-ALEXANDER; SINELNIKOV; CURTNER-SMITH, 2016).

Previous and current reviews showed that the SEM developed students' enjoyment and satisfaction, which favoured PE practise (MACPHAIL et al., 2008; KINCHIN; MACPHAIL; NI CHRÓINÍN, 2012; CUEVAS; GARCÍA-LÓPEZ; SERRA-OLIVARES, 2016) this is very important in amotivated students (PERLMAN, 2012). Moreover, responsibility fostered by the model's roles (i.e., coach, captain, referee or judge) seemed to influence students' intrinsic motivation (MARTíNEZ; MÉNDEZ-GIMÉNEZ; VALVERDE, 2016; ROMAR; SARÉN; HASTIE, 2016) through an increase in their self-determined competence (CUEVAS; GARCÍA-LÓPEZ; SERRA-OLIVARES, 2016; MENÉNDEZ-SANTURIO; FERNÁNDEZ-RÍO, 2016). Furthermore, students' responsibility increased when the SEM was hybridized with TPSR (MENÉNDEZSANTURIO; FERNÁNDEZ-RÍO, 2016b).

Finally, SEM not only increased students' positive personal and social values (i.e., assertiveness, cooperation or empathy) (GARCÍA-LÓPEZ; GUTIÉRREZ, 2013; MARTÍNEZ; MÉNDEZ-GIMÉNEZ; VALVERDE, 2016; ROMAR; SARÉN; HASTIE, 2016), but it also increased aggressiveness when the implementation was conducted using invasion games (GARCÍA-LÓPEZ; GUTIÉRREZ, 2013), specifically with some roles performed by the students (i.e., coach or technical director). On the other hand, the SEM can also help reduce negative behaviours such as students' attitudes towards violence and aggressiveness (MENÉNDEZSANTURIO; FERNÁNDEZ-RÍO, 2016b) when it is hybridized with TPSR and implemented using combat sports. Students' physical self-concept (FERNÁNDEZ-RÍO; MÉNDEZ-GIMÉNEZ; MÉNDEZ-ALONSO, 2013), intention to be physically active (MARTÍNEZ; MÉNDEZ-GIMÉNEZ; VALVERDE, 2016) and emotional intelligence (MÉNDEZ-GIMÉNEZ; MARTÍNEZ; VALVERDE, 2017) were novel research areas not included in previous reviews.

\subsection{Game performance/tactical knowledge: cognitive and physical learning outcomes}

Cognitive and physical learning outcomes of PE are connected to game performance and tactical knowledge. Cognitive learning was the outcome most frequently assessed (39, $47 \%$ ), while physical learning was assessed in fewer studies (13,16\%). Previous and current reviews showed that the SEM favoured students' game performance and tactical knowledge through an increase in their competence (PRITCHARD et al., 2008; HASTIE; SINELNIKOV; GUARINO, 2009; CALDERÓN; MARTÍNEZ; HASTIE, 2013); specially, when SEM was 
hybridized with other models such as Teaching Games for Understanding (TGFU) (HASTIE; CURTNER-SMITH, 2006), or the Invasion Games Competence Model (IGCM) (FARIAS; MESQUITA; HASTIE, 2015). However, such a complex framework can also cause negative outcomes in the students' understanding of tactical principles (GUTIÉRREZ et al., 2014).

Some studies highlighted that SEM can increase students' skill level, perceived competence and tactical knowledge (PRITCHARD et al., 2008; PEREIRA et al., 2015), as well as their participation and enthusiasm (CALDERÓN; MARTÍNEZ; MARTíNEZ, 2013) more than other instructional framework such as DI. Nonetheless, some studies showed similar improvements in sports learning in both structures (HASTIE et al., 2013; PEREIRA et al., 2016). When considering students' skill level and the effects of the SEM in their improvements, research has been inconclusive: (1) less-participant students increased motivation and inclusion (PILL, 2008; PERLMAN, 2012); (2) medium-level students had bigger improvements (MAHEDERO et al., 2015); and (3) boys and girls of all skill levels improved (PEREIRA et al., 2015). In all cases, high skill level or leader students seemed to play a very important role to help other students (HASTIE; CURTNER-SMITH, 2006; CALDERÓN; MARTÍNEZ; MARTÍNEZ, 2013).

Regarding gender, studies included in previous reviews showed that girls obtained higher levels of success, emphasizing the length of the seasons as an essential factor in these results (CARLSON 1995). In accordance with this finding, more recent research emphasised the improvements in skill execution and tactical decisions in girls (MESQUITA; FARIAS; HASTIE, 2012). Nevertheless, other studies found better results in skill development and tactical knowledge in boys (HASTIE; SINELNIKOV; GUARINO, 2009). Furthermore, girls showed more commitment with the roles (GARCÍA-LÓPEZ et al., 2012) and developed higher levels of responsibility and autonomy (FERNÁNDEZ-RÍO; MENÉNDEZ-SANTURIO, 2017). Finally, other studies showed less participation opportunities for boys (GUTIÉRREZ et al., 2013; HASTIE; WARD; BROCK, 2017). Again, results are contradictory and inconclusive.

\section{CONCLUSIONS}

The present review of the SEM literature from 2013 to 2017 showed that its implementation has expanded over the last five years to include all school grade levels and all continents to assess its impact in the four learning outcomes of PE (physical, social, cognitive and affective), and to join forces with other pedagogical frameworks to maximize their effects. SEM implementations have showed positive impacts on participants' learning (game performance and tactical-technical knowledge) regardless of their skill level and gender, in their social skills and values (empathy, assertiveness or fair play), and in their enthusiasm, enjoyment and preference for practising sports with this model. SEM also helped fulfil students' basic psychological needs, developing more competence, autonomy and relatedness. Finally, it is necessary to examine the possibilities of the SEM with other pedagogical models because it is a new trend of research. 


\section{REFERENCES}

ALEXANDER, Ken; LUCKMAN, Jan. Australian teacher's perceptions and uses of the Sport Education curriculum model. European Physical Education Review, v. 7, p. 243-267, 2001.

ARAÚJO, Rui et al. The evolution of student-coach's pedagogical content knowledge in a combined use of Sport Education and the Step-Game-Approach model. Physical Education and Sport Pedagogy, v. 22, n. 5, p. 518-535, 2017.

ARAÚJO, Rui; MESQUITA, Isabel; HASTIE, Peter Andrew. Review of the status of learning in research on Sport Education: future research and practice. Journal of Sports Science and Medicine, v. 13, p. 846-858, 2014.

ARAÚJO, Rui et al. Content knowledge of the student-coach in peer teaching tasks in a hybrid SESGA volleyball unit. Revista Mineira de Educação Física, v. 9, p. 49-55, 2013.

CALDERÓN, Antonio; HASTIE, Peter Andrew; MARTíNEZ, Diego. Aprendiendo a enseñar mediante el Modelo de Educación Deportiva (Sport Education): experiencia inicial en Educación Primaria. Cultura, Ciencia y Deporte, v. 15, p. 169-180, 2010.

CALDERÓN, Antonio; MARTíNEZ, Diego; HASTIE, Peter Andrew. Students and teachers' perception after practice with two pedagogical models in Physical Education. RICYDE. Revista Internacional de Ciencias del Deporte, v. 32, n. 9, p. 137-153, 2013.

CALDERÓN, Antonio, MARTíNEZ, Diego; MARTÍNEZ, Isabel. Influence of perceived skill on the attitude of students after a Sport Education unit. Retos, v. 24, p. 16-20, 2013.

CALDERÓN, Antonio et al. Now we help ourselves more: Team-teaching and social classroom climate. Experience with Sport Education. RICYDE. Revista Internacional de Ciencias del Deporte, v. 12, n. 44, p. 121-136, 2016.

CARLSON, Teresa. Now I think I can: The reaction of eight low-skilled students to sport education. ACHPER Healthy Lifestyles Journal, v. 42, p. 6-8, 1995.

CHONG, Swee; PENNEY, Dawn. Promoting social and emotional learning outcomes in physical education: Insights from a school-based research project in Singapore. Asia-Pacific Journal of Health, Sport and Physical Education, v. 4, n. 3, p. 267-286, 2013.

CUEVAS, Ricardo; GARCÍA-LÓPEZ, Luis Miguel; CONTRERAS, Onofre. Influence of the Sport Education Model in the psychological basic needs. Cuadernos de Psicología del Deporte, v. 15, n. 2, p. 155-162, 2015.

CUEVAS, Ricardo; GARCÍA-LÓPEZ, Luis Miguel; SERRA-OLIVARES, Jaime. Sport Education Model and self-determination theory: an intervention in secondary school children. Kineziologija, v. 48, n. 1, p. 30-38, 2016.

EVANGELIO, Carlos et al. The Sport Education Model in Spain: a review of state of the art and outlook. Cuadernos de Psicología del Deporte, v. 16, n. 1, p. 307-324, 2016.

FARIAS, Claudio; MESQUITA, Isabel; HASTIE, Peter Andrew. Game Performance and Understanding within a hybrid Sport Education season. Journal of Teaching in Physical Education, v. 34, n. 3, p. 363-383, 2015. 
FERNÁNDEZ-RÍO, Javier. Another step in Models-based practice: hybridizing Cooperative Learning and Teaching for Personal and Social Responsibility. Journal of Physical Education, Recreation and Dance, v. 85, p. 3-5, 2014.

FERNÁNDEZ-RÍO, Javier; MÉNDEZ-GIMÉNEZ, Antonio; MÉNDEZ-ALONSO, David. Effects of the three instructional approaches in adolescents' physical self-concept. Cultura y Educación, v. 25, n. 4, p. 509-521, 2013.

FERNÁNDEZ-RÍO, Javier, MÉNDEZ-GIMÉNEZ, Antonio and MÉNDEZ-ALONSO, David. Effects of two instructional approaches, Sport Education and Direct Instruction, on secondary education students' psychological response. SPORT TK-Revista EuroAmericana de Ciencias del Deporte, v. 6, n. 2, 9-20, 2017.

FERNÁNDEZ-RÍO, Javier; MENÉNDEZ-SANTURIO, José Ignacio. Teachers and students' perceptions of a hybrid Sport Education and Teaching for Personal and Social Responsibility learning unit. Journal of Teaching in Physical Education, v. 36, n. 2, p. 185-196, 2017.

GARCÍA-LÓPEZ, Luis Miguel; GUTIÉRREZ, David. The effects of a sport education season on empathy and assertiveness. Physical Education and Sport Pedagogy, v. 14, p. 355-375, 2013.

GARCÍA-LÓPEZ, Luis Miguel et al. Changes in empathy, assertiveness and social relations due to the implementation of the Sport Education Model. Revista de Psicología del Deporte, v. 2, p. 321-330, 2012.

GIL-ARIAS, Alexander et al. Impact of a hybrid TGfU-Sport Education unit on student motivation in physical education. PLoS ONE, v. 12, n. 6, p. 1-17, 2017.

GINCIENE, Guy; MATTHIESEN, Sara Quenzer. The Sport Education Model on the teaching of track and field in school. Movimento, v. 23, n. 2, p. 729-742, 2017.

GUTIÉRREZ, David et al. Sport Education Model in second grade. Teachers and student's perceptions. Cuadernos de Psicología del Deporte, v. 14, n. 2, p. 131-144, 2014.

GUTIÉRREZ, David et al. The responses of Spanish students' to participation in seasons of sport education. The Global Journal of Health and Physical Education Pedagogy, v. 2, n. 2, p. 111-127, 2013.

HASTIE, Peter Andrew et al. The development of skill and knowledge during a sport education season of track and field athletics. Research Quarterly for Exercise and Sport, v. 84, n. 3, p. 336$344,2013$.

HASTIE, Peter Andrew; CURTNER-SMITH, Matthew. Influence of a hybrid Sport Education: Teaching Games for Understanding unit on one teacher and his students. Physical Education and Sport Pedagogy, v. 11, n. 1, p. 1-27, 2006.

HASTIE, Peter Andrew et al. Perceived and actual motivational climate of a mastery-involving sport education season. European Physical Education Review, v. 20, n. 2, p. 215-228, 2014.

HASTIE, Peter Andrew; FARIAS, Claudio; GUTIÉRREZ, David. Students' and teachers' responses to a transatlantic sport education league. Journal of the International Society for Comparative Physical Education and Sport, v. 35, n. 2, p. 22-33, 2013.

HASTIE, Peter Andrew; MARTÍNEZ, Diego; CALDERÓN, Antonio. A review of research on Sport Education: 2004 to the present. Physical Education and Sport Pedagogy, v. 16, p. 103-132, 2011. 
HASTIE, Peter Andrew; SINELNIKOV, Oleg; GUARINO, Anthony. The development of skill and tactical competencies during a season of badminton. European Journal of Sport Science, v. 9, n. 3, p. 133140, 2009.

HASTIE, Peter Andrew; WALLHEAD, Tristan. Models-Based Practice in Physical Education: The Case for Sport Education. Journal of Teaching in Physical Education, v. 35, p. 390-399, 2016.

HASTIE, Peter Andrew; WARD, Kurt; BROCK, Sheri. Effect of graded competition on student opportunities for participation and success rates during a season of Sport Education. Physical Education and Sport Pedagogy, v. 22, n. 3, p. 316-327, 2017.

KINCHIN, Gary, A; MACPHAIL, Ann; NI CHRÓINÍN, Déirdre. Irish primary school teachers' experiences with Sport Education. Irish Educational Studies, v. 31, n. 2, p. 207-222, 2012.

KIRK, David. Educational value and models-based practice in physical education. Educational Philosophy and Theory, v. 45, n. 9, p. 973-986, 2013.

LAYNE, Todd; HASTIE, Peter Andrew. A task analysis of a Sport Education physical education season for fourth grade students. Physical Education and Sport Pedagogy, v. 20, n. 3, p. 314-328, 2013.

LAYNE, Todd; HASTIE, Peter Andrew. Competition within Physical Education: using Sport Education and other recommendations to create a productive, competitive environment. Strategies, v. 27, n. 6, p. 3-7, 2014a.

LAYNE, Todd; HASTIE, Peter Andrew. Analysis of teaching Physical Education to second-grade students using Sport Education. International Journal of Primary, Elementary and Early Years Education, v. 44, n. 2, p. 226-240, 2014b.

MACPHAIL, Ann et al. Children's experiences of fun and enjoyment during a season of Sport Education. Research Quarterly for Exercise and Sport, v. 79, n. 3, p. 344-355, 2008.

MACPHAIL, Ann; KINCHIN, Gary. The use of drawings as an evaluative tool: Students' experiences of sport education. Physical Education and Sport Pedagogy, v. 9, n. 1, p. 87-108, 2004.

MAHEDERO, Pilar et al. Effects of student skill level on knowledge, decision making, skill execution and game performance in a mini-volleyball Sport Education season. Journal of Teaching in Physical Education, v. 34, n. 4, p. 626-641, 2015.

MANTEROLA, Carlos et al. Systematic reviews of the literature: what should be known about them. Cirugía Española (English Edition), v. 91, n 3, p. 149-155, 2013.

MARTÍNEZ, Diego; MÉNDEZ-GIMÉNEZ, Antonio; VALVERDE, Juan José. Sport Education Model effects in the social climate classroom, perceived competence and intent to be physically active: an extended study in Primary Education. SPORT TK-Revista EuroAmericana de Ciencias del Deporte, v. 5, n. 2, p. 153-166, 2016.

MÉNDEZ-GIMÉNEZ, Antonio; FERNÁNDEZ-RíO, Javier; MÉNDEZ-ALONSO, David. Sport Education Model versus traditional model: effects on motivation and sportsmanship. Revista Internacional de Medicina y Ciencias de la Actividad Física y el Deporte, v. 15, n. 59, p. 449-466, 2015.

MÉNDEZ-GIMÉNEZ, Antonio; MARTíNEZ, Diego. Percepciones del estudiante de primaria sobre el uso del modelo de Educación Deportiva para la enseñanza del mimo. Revista Española de Educación Física y Deportes, v. 418, n. 3, p. 21-34, 2017. 
MÉNDEZ-GIMÉNEZ, Antonio; MARTÍNEZ, Diego; VALVERDE, Juan José. Student and teachers' assessment of conventional and self-made material: longitudinal crossover study in Physical Education. Retos, v. 30, p. 20-25, 2016.

MÉNDEZ-GIMÉNEZ, Antonio; MARTÍNEZ, Diego; VALVERDE, Juan José. Inteligencia emocional y mediadores motivacionales en una temporada de Educación Deportiva sobre mimo. Ágora para la Educación Física y el Deporte, v. 19, n. 1, p. 52-72, 2017.

MENÉNDEZ-SANTURIO, José Ignacio; FERNÁNDEZ-RÍO, Javier. Hybridizing Sport Education and Teaching for Personal and Social Responsibility: an experience through an educational kickboxing program. Retos, v. 30, p. 113-121, 2016a.

MENÉNDEZ-SANTURIO, José Ignacio; FERNÁNDEZ-RÍO, Javier. Violencia, responsabilidad, amistad y necesidades psicológicas básicas: efectos de un programa de Educación Deportiva y Responsabilidad Personal y Social. Revista de Psicodidáctica, v. 21, n. 2, p. 245-260, 2016b.

MENÉNDEZ-SANTURIO, José Ignacio; FERNÁNDEZ-RÍO, Javier. Hybridizing Sport Education and Teaching for Personal and Social Responsibility to include students with disabilities. European Journal of Special Needs Education, v. 34, n. 2, p. 508-524, 2017.

MESQUITA, Isabel; FARIAS, Claudio; HASTIE, Peter Andrew. The impact of a hybrid Sport EducationInvasion Games Competence Model soccer unit on students' decision making, skill execution and overall game performance. European Physical Education Review, v. 18, p. 205-219, 2012.

PEREIRA, José et al. Sport Education and Direct Instruction Units: comparison of student knowledge development in athletics. Journal of Sports Science and Medicine, v. 15, n. 4, p. 569-577, 2016.

PEREIRA, José et al. A Comparative Study of Students' Track and field technical performance in Sport Education and in a Direct Instruction approach. Journal of Sports Science and Medicine, v. 14, n. 1, p. 118-127, 2015.

PERLMAN, Dana. An examination of amotivated students within the sport education model. AsiaPacific Journal of Health: Sport and Physical Education, v. 3, n. 2, p. 141-155, 2012.

PILL, Shane. A teacher's perceptions of the Sport Education Model as an alternative for upper primary school physical education. ACHPER Australia Healthy Lifestyles Journal, v. 55, n. 2, p. 23-29, 2008.

PRITCHARD, Tony et al. Effects of two instructional approaches on skill development, knowledge, and game performance. Measurement in Physical Education and Exercise Science, v. 12, n. 4, p. 219236, 2008.

QUILL, Mandy; CLARKE, Gill. Sport Education in gymnastics. In: PENNEY, Dawn et al. Sport Education in Physical Education: research based practice. New York: Routledge, 2005. p. 165-175.

ROMAR, Jan Erik; SARÉN, Jani; HASTIE, Peter Andrew. Athlete-Centred coaching using the Sport Education Model in youth soccer. Journal of Physical Education and Sport, v. 16, n. 2, p. 380-391, 2016.

SIEDENTOP, Daryl; HASTIE, Peter Andrew; VAN DER MARS, Hans. Complete Guide to Sport Education. 2. ed. Champaign: Human Kinetics, 2011.

SINELNIKOV, Oleg. Sport education for teachers: Professional development when introducing a novel curriculum model. European Physical Education Review, v. 15, n. 1, p. 91-114, 2009. 
VLACHOPOULOS, Symeon; MICHAILIDOU, Sotiria. Development and initial validation of a measure of autonomy, competence, and relatedness in exercise: The Basic Psychological Needs in Exercise Scale. Measurement in physical education and exercise science, v. 10, n. 3, p. 179-201, 2006.

WAHL-ALEXANDER, Zachary; SINELNIKOV, Oleg; CURTNER-SMITH, Matthew. A longitudinal analysis of students' autobiographical memories of participation in multiple Sport Education seasons. European Physical Education Review, v. 23, n. 1, p. 25-40, 2016.

WALLHEAD, Tristan; O'SULLIVAN, Mary. Sport education: physical education for the new millennium? Physical Education and Sport Pedagogy, v. 10, n. 2, p. 181-210, 2005.

WARD, Kurt et al. A Sport Education Fitness Season's impact on students' fitness levels, knowledge, and in-class physical activity. Research Quarterly for Exercise and Sport, v. 88, n. 3, p. 346-351, 2017. 